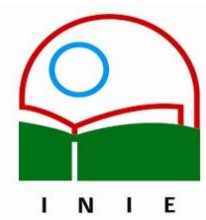

Actualidades Investigativas en Educación

Revista Electrónica publicada por el

Instituto de Investigación en Educación

Universidad de Costa Rica

ISSN 1409-4703

http://revista.inie.ucr.ac.cr

COSTA RICA

\title{
NOSOTROS Y LOS OTROS. ¿CÓMO SE REPRESENTAN LOS NIÑOS Y LAS NIÑAS LAS NORMAS SOCIALES?
}

US AND OTHERS. HOW DO BOYS AND GIRLS REPRESENT SOCIAL NORMS?

\author{
Volumen 9, Número 3
}

pp. 1-29

Este número se publicó el 15 de diciembre 2009

Ana Rebeca Urmeneta Garrido

La revista está indexada en los directorios:

LATINDEX, REDALYC, IRESIE, CLASE, DIALNET, DOAJ, E-REVIST@S,

La revista está incluida en los sitios:

REDIE, RINACE, OEI, MAESTROTECA, PREAL, HUASCARAN, CLASCO 


\title{
NOSOTROS Y LOS OTROS. ¿CÓMO SE REPRESENTAN LOS NIÑOS Y LAS NIÑAS LAS NORMAS SOCIALES? \\ US AND OTHERS. HOW DO BOYS AND GIRLS REPRESENT SOCIAL NORMS?
}

\author{
Ana Rebeca Urmeneta Garrido ${ }^{1}$
}

\begin{abstract}
Resumen: Los resultados presentados en este texto son el fruto de una investigación cualitativa sobre el mundo social infantil. Su principal objetivo es conocer y comprender las representaciones sociales infantiles sobre las normas y la cohesión social. El artículo reflexiona sobre las siguientes cuestiones: ¿Cómo definen la norma social los niños y las niñas? ¿En qué valores y motivos la fundamentan? ¿Cómo se sostiene el orden y la cohesión social desde la perspectiva infantil? La metodología de investigación se basa en una técnica cualitativa innovadora y de elaboración propia: el Cuento Inconcluso. Se trata de un relato inacabado en el que se plantea un conflicto normativo (la desobediencia a una norma social) y que los participantes deben desarrollar y finalizar. Estudiamos una muestra de 150 relatos, escritos por estudiantes de 8 a 14 años de diez escuelas de la ciudad de Barcelona (España), 3 de nivel socioeconómico medio-alto y 7 de nivel bajo. El análisis estructural de contenido de estos textos revela la existencia de tres modelos básicos de representación social infantil de la norma: 1) la norma como núcleo de la identidad y la pertenencia a la comunidad; 2) la norma como salvaguardia del bien común; y 3) la norma como forma de racionalidad y madurez. Comentamos las principales características de estos modelos e ilustramos el análisis con algunos casos concretos.
\end{abstract}

Palabras clave: INFANCIA, NORMAS SOCIALES, COHESIÓN SOCIAL, IMAGINARIO SOCIAL, ANÁLISIS ESTRUCTURAL DE CONTENIDO, CUENTOS INFANTILES

\begin{abstract}
The findings exposed in this paper are the outcome of a qualitative study into the social world of children. Its main objective is to identify and comprehend children's social representations of norms and social cohesion. The article seeks answers to the following questions: How do boys and girls define the social norm? What values and motives do they base it on? How are order and social cohesion upheld from a children's point of view? The research methodology is based on a novel qualitative technique we developed ourselves: the Unfinished Story. It consists of an unfinished story that presents a normative conflict (disobedience of a social norm) and the participants are then asked to write an ending to the story. We studied a sample of 150 stories written by 896 students aged 8 to 14 from ten schools in the city of Barcelona (Spain), 3 were upper-middle class schools and 7 were lower class schools. A structural analysis of the contents of these stories revealed that children's representations of the social norm could be categorised into three basic models: 1) the norm as the core of identity and belonging to the community; 2) the norm as a safeguard of the common good; and 3) the norm as a form of rationality and maturity. We then go on to expound on the main features of these models and illustrate our analysis with a series of concrete examples.
\end{abstract}

Keywords: CHILDHOOD, SOCIAL NORMS, SOCIAL COHESION, SOCIAL IMAGINARY, STRUCTURAL ANALYSIS OF CONTENTS, CHILDREN'S STORIES

\footnotetext{
1 Doctora en Sociología, Profesora de la Universidad de Girona (España), Facultad de Ciencias Económicas y Empresariales. Departamento de Empresa. Investigadora del Observatorio del Desarrollo Humano de Cataluña.
}

Dirección electrónica: ana.urmeneta@udg.edu

Artículo recibido: 4 de marzo, 2009

Aprobado: 30 de noviembre, 2009 


\section{Introducción}

En la actualidad, existen muy pocos estudios sociológicos sobre cómo la infancia ve a la sociedad. Las Ciencias Sociales, y en especial la Sociología, se han caracterizado tradicionalmente por ser adultocéntricas. Se ha minimizado la importancia de la investigación sobre el mundo social de la infancia desde el propio sujeto infantil, relegando a los niños y niñas a un papel pasivo, como meros objetos o recipientes, negando o restando, en muchas ocasiones, su potencial de acción (Jenks, 1982). Se ha tendido a confundir a los menores con seres pre-sociales o agentes sociales incompletos, subestimando, de esta forma, su capacidad para interpretar, construir e influir de manera activa en el mundo social que les rodea. El presente artículo busca paliar en alguna medida esta carencia. Exponemos los resultados más relevantes de una novedosa investigación educativa sobre el imaginario social infantil, correspondiente a nuestra tesis doctoral: Reificación, Reproducción y Resistencia al Mensaje Disciplinario de los Cuentos Infantiles (Urmeneta, 2008). El objetivo de nuestro estudio es analizar las representaciones infantiles sobre las normas sociales. La investigación fue realizada en la ciudad de Barcelona (España) y participaron menores entre 8 y 14 años de edad. Si bien los resultados de esta investigación se hallan referidos a ese contexto social, creemos que pueden ser extrapolados a otros; y aunque éstos den cuenta de la realidad específica de los niños y niñas catalanes, constituyen un indicio de cómo es vista la sociedad desde los ojos de la infancia, trascendiendo las fronteras y las particularidades nacionales.

Las representaciones sociales constituyen un conjunto de creencias, valores y prácticas compartidas por un determinado grupo social (Moscovici, 1985-1986). Este conocimiento no se produce individualmente sino dentro de un determinado contexto. En su interacción con los otros, el sujeto construye una imagen de sí mismo y de la sociedad en la que se halla inserto. Se trata, por tanto, de un conocimiento que los sujetos infantiles no poseen en sus "cabezas", como si fuera el fruto aislado de su subjetividad, sino de algo que hacen y construyen socialmente en su interacción cotidiana, tanto con los adultos como con sus pares (Gergen, 1993). En cierta forma, podríamos decir que las representaciones sociales se encuentran 'flotando' en las sociedades, impregnando todos los aspectos de nuestra vida social. Cumplen dos importantes funciones: 1) establecen un orden que permite a los individuos orientarse en su mundo material y social, y 2) posibilitan la comunicación entre los miembros de una comunidad, proporcionándoles un código para el intercambio social y un Volumen 9, Número 3, Año 2009, ISSN 1409-4703 
código para nombrar y clasificar, sin ambigüedades, los diversos aspectos de su mundo social, su historia individual y grupal (Farr, 1983).

En el presente artículo buscamos aproximarnos a la mirada sobre el Nosotros y los Otros que construye socialmente la infancia, a partir del análisis de su comprensión de la Norma, el Orden y la Cohesión Social. Este conocimiento colectivo infantil delimita su sentido de pertenencia a la sociedad, establece los límites de la tolerancia a la diferencia y determina los márgenes de posibilidad y de cambio social. Específicamente, nos preguntamos ¿Cómo definen la norma social los niños y las niñas? ¿En qué valores o motivos la fundamentan? ¿Cómo se sostiene el orden y la cohesión social desde la perspectiva infantil? Nuestra hipótesis sostiene que los sujetos infantiles interpretan activamente la realidad social en la que se hallan insertos. Construyen elaboradas teorías sobre el origen y la función de las normas sociales, y en forma general sobre la cohesión y el funcionamiento del orden social. Lo anterior denotaría la existencia de un pensamiento sociológico infantil. Dicho pensamiento no se construye individualmente, es un pensamiento colectivo, un conjunto de representaciones sociales sobre la sociedad y sus instituciones. Dicho contenido representacional puede ser develado mediante el análisis del discurso infantil.

\section{Referente Teórico y Conceptual}

Nuestro análisis e interpretación de las representaciones infantiles, toma como marco de referencia teórica algunos de los conceptos básicos de la disciplina sociológica. Tales como: norma, rol, orden social, núcleo social, cohesión social, identidad, imperativo institucional, conformidad, desviación y control social. Observamos una notable correspondencia entre dichos términos y la forma en que la infancia concibe la norma y la cohesión social, indicándonos, tal como señalamos antes, la existencia de un cierto pensamiento sociológico infantil. En esta sección exponemos brevemente el marco conceptual que empleamos más adelante, en la discusión de nuestros resultados.

Una norma es una regla, un patrón de medida o una pauta de acción (del latín norma: escuadra de carpintero o regla). Las normas sociales son reglas de conducta que representan una definición cultural del comportamiento socialmente deseable. Se trata del patrón según el cual comparamos y juzgamos el comportamiento y a tenor del cual le Volumen 9, Número 3, Año 2009, ISSN 1409-4703 
otorgamos nuestra aprobación o repulsa. Las normas expresan expectativas de roles, es decir: señalan la manera en la que deben comportarse los miembros de una formación social frente a los otros miembros (Hillmann, 2001; Sills, 1979).

Las normas dotan de sentido al mundo social. Por su referencia a una estructura de reglas o de normas colectivas, toda conducta humana es significativa y coherente, tanto a los ojos del propio actor como para aquellos con quienes o en medio de quienes éste actúa. Las normas otorgan patrones por medio de los cuales se modela la conducta humana, dentro de los canales que la sociedad considera como lo más conveniente. Las normas nos orientan (prescriben) y nos constriñen (proscriben); esto permite una cierta regularidad y una tipificación de las conductas que permite simplificar enormemente la vida cotidiana. Las expectativas de los demás sobre nosotros y de nosotros sobre los demás no dependen del azar, son predecibles en la medida en que se hallan socialmente reguladas y sujetas a normas.

El orden social, es decir, es decir el conjunto de normas, leyes y estructuras que definen y sitúan al sujeto en el mundo, constituye el núcleo de lo social, lo que Durkheim (1982 y 2001) denomina Lo Sagrado. En dicho núcleo se establecen y definen las distinciones básicas entre la noción del Nosotros y la noción de los Otros: los que compartimos una forma común de situarnos y relacionarnos con el mundo, es decir una identidad social, y los que se oponen de alguna manera a ésta y/o se hallan al margen de ella. El núcleo social es la base de la cohesión social; organiza y estructura nuestras percepciones acerca de las cosas, los acontecimientos y los demás sujetos en el mundo, otorgándonos un marco desde el cual interpretar/construir la realidad y conformar nuestra identidad y pertenencia en ella (Berger \& Luckmann, 1999). Dicho marco define y establece lo que socialmente ha de ser considerado, por los individuos pertenecientes a una determinada comunidad, como: 1) Lo real, trazando una línea divisoria entre lo que legítimamente existe y lo que no existe; 2) Lo normal, estableciendo distinciones entre lo bueno y lo malo, lo justo y lo injusto, lo racional y lo irracional, lo mayoritario y lo minoritario, etc. constituyendo de esta forma las categorías que enmarcan las representaciones sociales de lo normal y lo anormal; y, finalmente, 3) Lo posible, definiendo un marco que constriñe el sentido de cambio y abre/cierra nuestras posibilidades de pensar en un orden diferente (Thërborn, 1987). 
Este marco de referencia compartido conforma una cosmovisión o forma de ver y situarse en el mundo, que no sólo orienta las conductas de los individuos, sino que los construye como tales. Esta cosmovisión, tal como la hemos definido aquí constituye un proceso ideológico (Thërborn, 1987) y un imperativo institucional (Berger, 2002). Ahora bien, es preciso señalar que la relación del individuo con el orden social no es de determinación. El individuo construye su realidad en un contexto interactivo, tiene intenciones, interpreta las situaciones y les atribuye un significado. Las normas no nos determinan, pero sí nos proveen de los significados básicos de la vida social; nos otorgan el mapa desde el cual interpretamos y construimos el mundo y a Nosotros dentro de ese mundo (Berger, 2002). Representan por tanto una definición del Nosotros, de qué significa ser miembro de esta sociedad y de lo que se espera respecto a nuestro comportamiento dentro de ella.

La conformidad a la norma no es un acto pasivo. Si bien la conformidad supone la aceptación del orden social por parte del sujeto, no implica una relación inmediata entre éste y dicho orden. La relación entre individuo y sociedad se halla siempre mediada por la interpretación que el individuo hace del orden social en el que se inserta. El sujeto integra activamente el mundo normativo, y, por tanto, hay siempre posibilidades de innovación, sin que ello implique necesariamente la transgresión o la salida del sistema. La frontera que separa la innovación de la transgresión ${ }^{2}$ puede a veces parecer difusa, pero la sociedad establece mecanismos precisos para asegurar dicha distinción. En términos generales, se trata de la construcción social de la identidad y de la diferencia, lo que define al Nosotros y a los Otros, y lo que separa la conformidad, incluida también la innovación, de la transgresión. Estas dos nociones son indisolubles, no es posible entender la una sin la otra, pues ambas forman parte de una misma totalidad.

La sociedad emplea un conjunto de mecanismos para mantener a sus miembros dentro de los límites del orden social y para minimizar la influencia de quienes se apartan de éste. Estos mecanismos conforman el llamado Control Social, cuyo objetivo es neutralizar el comportamiento desviado: 1) impidiendo la aparición de tensiones que susciten el deseo de

${ }^{2}$ En sociología, se usa habitualmente el término desviación para referirse a las conductas que se apartan de las normas sociales, es decir las conductas anómicas. Nosotros hemos empleado el concepto de transgresión, quizás menos técnico, pero que a nuestro juicio se ajusta mejor al mundo representacional infantil. 
desviarse, éste es uno de los objetivos fundamentales del proceso de socialización; 2) reforzando el deseo de mantener la actitud de conformidad, es decir de ajuste o acomodación a las expectativas sociales ; 3) poniendo de manifiesto lo que es socialmente conveniente; 4) dificultando la desviación por medio de sanciones (positivas o negativas); y, 5) modificando las pautas sociales con el fin de encajar y asimilar, en la medida de lo posible, el comportamiento desviado de los actores (Hillmann, 2001 y Sills, 1979).

\section{Metodología}

El estudio presentado en este texto forma parte de una investigación mixta de carácter más amplio que la aquí reseñada (Urmeneta, 2008). En ella se emplearon tanto técnicas cuantitativas como cualitativas, y se realizó un proceso de triangulación en el análisis (Arias, 1999). La perspectiva metodológica principal de dicho estudio fue la cualitativa: QI-qt (Bericat, 1998). La investigación fue llevada a cabo en diez escuelas de la ciudad de Barcelona (España): 5 públicas, 2 concertadas $^{3}$ y 3 privadas. Participaron 896 menores con edades comprendidas entre los 8 y los 14 años, estudiantes de $4^{\circ}$ de Enseñanza Primaria y $2^{\circ}$ de Enseñanza Secundaria Obligatoria. Los resultados presentados en este artículo, corresponden al análisis cualitativo de una submuestra de 150 relatos ${ }^{4}$.

Es muy importante destacar que nuestro análisis sobre los cuentos infantiles, no se centra únicamente en la descripción de los contenidos de los mismos (los conceptos empleados), sino también en cómo éstos son integrados dentro de la estructura del texto. Hemos aplicado a los cuentos escritos por los menores la técnica del Análisis Estructural del Contenido (Hiernaux, 1977; Remy \& Ruquoy, 1990) ${ }^{5}$. Nuestro propósito es estudiar las complejas

${ }^{3}$ Las escuelas concertadas son centros privados que mantienen un concierto con la Administración Pública que paga a sus profesores y subvenciona su funcionamiento. Se han de atener a la misma legislación, calendarios, programas y organización que los colegios públicos. Teóricamente, las etapas subvencionadas (las que son obligatorias) han de ser gratuitas. En la práctica, suelen tener ciertos costes mensuales vinculados a actividades complementarias, clases extraescolares $u$ otros conceptos. Muchos de estos centros son católicos, aunque también los hay sin adscripción religiosa. Las instalaciones suelen ser buenas y los programas extracurriculares variados. Por lo general, estos establecimientos se hayan dirigidos a estudiantes de clases medias.

${ }^{4}$ Algunos de los textos presentados en este artículo fueron escritos en catalán, aquí presentamos su traducción al castellano. Hemos procurado respetar la ortografía y redacción de los textos originales.

${ }^{5}$ Existen muchas otras formas de análisis estructural del cuento. Ver por ejemplo los trabajos de Greïmas (1983) y Propp (1985). 
cadenas asociativas subyacentes en los textos y desvelar la función que dichas estructuras tienen dentro del sistema de representación social infantil de la norma. A efectos metodológicos, esto supone analizar las secuencias completas de los relatos, y no segmentos aislados; pues es en dichas estructuras en donde se expresa con mayor claridad la concepción de la infancia sobre la norma y el orden social. Nuestra unidad de análisis, por tanto, es el cuento en su totalidad, y no secciones del mismo, como podría ser el caso de otro tipo de estudios. Sin embargo, en el presente artículo incluimos únicamente fragmentos de los textos originales. Hemos tomado esta decisión debido principalmente a las limitaciones de espacio, pero también con el objetivo de simplificar tanto la redacción como la lectura del texto.

\subsection{Diseño y Muestra}

Se construyó una muestra no probabilística basada en perfiles teóricos (casos tipo). Se aplicaron dos criterios de selección: el nivel socioeconómico de las familias (familias de nivel medio-alto y bajo) y la ideología religiosa de los centros (escuelas católicas y laicas). Como indicador del nivel socioeconómico se empleó el I. C. E. F. (Índice de capacidad económica familiar), elaborado por el Institut d'Estadística del Ayuntamiento de Barcelona. Mediante este indicador se identificó y escogió los barrios más pobres y ricos de la ciudad: Nou Barris ${ }^{6}$, y Sarrià-Sant Gervasi y Les Corts-Pedralbes, respectivamente. Para la elección de las escuelas católicas y laicas tipo, se emplearon listas especializadas elaboradas por la Generalitat de Catalunya (Departament d'Ensenyament), el Secretariat de l'Escola Cristiana de Catalunya, y los datos proporcionados por informantes clave. De esta forma, se construyó la muestra final, conformada por 3 escuelas de nivel socioeconómico alto (una de ellas católica) y 7 de nivel bajo (2 de ellas católicas). Se decidió incluir un número mayor de escuelas de sectores populares para solventar los problemas derivados de las dificultades lecto-escritoras presentes en algunos estudiantes de dicho estrato social; hecho que podría afectar la extensión y coherencia de los textos obtenidos.

${ }^{6}$ Decidimos excluir de nuestra muestra al barrio de Ciutat Vella, que si bien es uno de los más pobres de Barcelona, cuenta con altas tasas de inmigración, una variable interviniente que no podíamos controlar apropiadamente. Por esta razón, escogimos a Nou Barris para representar el nivel socioeconómico bajo, que a pesar de ser uno de los barrios más degradados y populares de Barcelona, presenta una tasa de inmigración similar a la del resto de la ciudad. 


\subsection{Instrumentos y procedimientos}

El estudio de las representaciones sociales de la infancia constituye un objeto de interés sociológico y educativo de primer orden. Permite reconocer los modos y procesos que organizan el pensamiento social infantil y estudiar cómo construyen y cómo son construidos por la realidad social. En la actualidad, existe una escasez de estudios sociológicos acerca del imaginario social infantil. Esta situación se debe principalmente a las dificultades metodológicas que comporta este objeto de investigación. La cuestión sobre cómo estudiar la infancia como actor social, supone tres importantes desafíos metodológicos derivados de tres tipos de problemas: a) las limitaciones en cuanto a las capacidades cognitiva y discursiva de los menores; b) la posición asimétrica que ocupa la infancia frente a la persona adulta que investiga; y en general, c) la consideración social de la población infantil como un grupo tutelado, al cual sólo se puede acceder con el consentimiento previo de las personas adultas de su entorno (Rodríguez Pascual, 2006, p. 67).

Con el fin de conocer y describir el mundo social infantil, y solventar los problemas metodológicos anteriormente expuestos, se diseñó una técnica cualitativa de carácter proyectivo: el Cuento Inconcluso. Esta herramienta de investigación ha sido probada y empleada con éxito en dos estudios previos (Urmeneta, 2004, 2008, 2009 abril y junio 26 a y b) sobre la representación social infantil y adolescente de diversos objetos sociales. Consiste en presentar a los participantes el inicio de un relato en el que se expone un conflicto normativo (la desobediencia del personaje principal a una norma importante para la comunidad); a continuación se les solicita que escriban el final de la historia. El Cuento Inconcluso presenta algunos aspectos comunes con la técnica de Dilemas Normativos (Kohlberg, 1992; Kohlberg, Power, \& Higgins, 2008) empleada en el estudio del desarrollo del juicio moral infantil. No obstante, es preciso destacar las diferencias existentes entre ambas herramientas, en cuanto a su objeto de estudio (desarrollo moral v/s representaciones sociales de la norma); su sustento teórico conceptual (psicología del desarrollo v/s sociología de la norma y el control social); y sus procedimientos de aplicación (entrevista personal o grupal v/s redacción individual) y de análisis (interpretación psicológica v/s estructural de contenido).

El texto que empleamos en el Cuento Inconcluso hacía referencia a una norma relacionada con el medio ambiente, que según los docentes entrevistados constituía un tema de gran Volumen 9, Número 3, Año 2009, ISSN 1409-4703 
interés para los menores. Se construyeron dos versiones del instrumento: un relato ambientado en un escenario urbano (parques de una ciudad turística) y otro en un ambiente rural (bosques de una pequeña localidad). La actividad fue desarrollada en una única sesión de aproximadamente 45 minutos. El análisis de los textos redactados por los estudiantes, permitió desvelar los sistemas de significación y los modelos culturales subyacentes en los textos, así como la identificación y reconstrucción de las representaciones sociales en torno a la norma en la infancia.

La técnica Cuento Inconcluso, se ajustó apropiadamente a las capacidades cognitivas y discursivas de los menores, obteniéndose textos de gran calidad y complejidad. Fue aplicada con éxito, tanto en los estudiantes de $4^{\circ}$ de Enseñanza Primaria como en los de $2^{\circ}$ de Enseñanza Secundaria Obligatoria, aunque con mejores resultados en este último grupo. La mayoría de los participantes desarrolló la actividad sin problemas y no necesitó aclaraciones posteriores. El conflicto normativo presentado al inicio del cuento dejó un margen suficientemente amplio de respuesta; lo que quedó reflejado, posteriormente, en la gran variedad de finales obtenidos. El carácter proyectivo y lúdico de la técnica permitió acortar la brecha entre la persona adulta que investiga y la infancia, como sujeto de investigación. Se procuró, en todo momento, otorgar a los menores el protagonismo de la actividad, dándoles a entender que ellos y ellas se debían adueñar del relato. Algunos participantes se entusiasmaron al tal punto con esta idea, que consignaron dicha autoría firmando sus trabajos. También hubo quienes decidieron incluir dibujos alusivos a sus cuentos.

En conclusión, si bien el acceso a los menores tuvo que pasar, necesariamente, por la autorización de las personas adultas a cargo (la dirección, el plantel docente y las familias); una vez superada esta primera barrera, se logró entrar en el mundo subjetivo infantil, descrito desde sus propios ojos y con sus propias palabras.

\section{Resultados y Discusión}

Hemos encontrado tres grandes ejes estructuradores de la representación social de la norma en el pensamiento infantil: el primero se halla centrado en la Identidad y Pertenencia al grupo, el segundo en el Bien Común, y, finalmente, el tercero en el respeto a la norma como Forma de Madurez y Racionalidad. Expondremos brevemente las principales características de estos tres modelos, los que serán ilustrados mediante algunos de los textos escritos por Volumen 9, Número 3, Año 2009, ISSN 1409-4703 
nuestros participantes. Hemos de entender estos modelos como guías que nos han permitido ordenar nuestro proceso de análisis y no como casillas cerradas y excluyentes. Muchos de los textos que hemos analizado encajan en más de una categoría al mismo tiempo, aunque exista en ellos una forma de representación predominante sobre la cual se construye el relato. Nuestro proceso analítico no ha estado orientado tanto a la categorización de los textos o a la construcción de una tipología, sino que a la comprensión/aprehensión de la lógica subyacente en ellos. Los modelos operan muchas veces de manera conjunta y compleja. La norma se construye en oposición a la transgresión y viceversa, y deben ser entendidas como las dos caras de una misma moneda.

\subsection{La Norma como fundamento de la Identidad y la Pertenencia a la Comunidad}

La norma es representada en la infancia como el fundamento primordial de la comunidad, aquello que nos cohesiona como Nosotros y nos proporciona una identidad propia y diferenciada. La transgresión, por su parte, es entendida como la destrucción del Contrato Social, y es representada en los relatos por la figura del Otro, la persona diferente ${ }^{7}$. Esta concepción de la transgresión no sólo se asocia a la idea de ruptura; los cuentos escritos por los menores indican que ésta cumple también una importante función social: la legitimación de las normas. Los relatos infantiles analizados presentan una notable correspondencia con la idea de funcionalidad del delito descrita por Durkheim (2001). Coincidiendo con los planteamientos de este autor, observamos en los cuentos de los menores que el efecto de distinción, categorización y aislamiento de las conductas consideradas como transgresoras, reafirma a toda la sociedad y a su centro ideológico básico, definido por Durkheim (1982) como Lo Sagrado. Este núcleo social permite diferenciar a los individuos adaptados de los desadaptados. De esta manera, se conforma un centro homogéneo y cohesionado en torno a la noción del Nosotros, cuyo opuesto es la periferia, siempre más heterogénea y disgregada, y que se expresa en forma patente en la incertidumbre y el miedo al Otro, aspectos claramente reflejados en los relatos infantiles. La distinción entre Nosotros y los

\footnotetext{
7 Hemos hallado tres formas de representación de la transgresión en los relatos infantiles: la transgresión como anormalidad, como incivilidad y como irracionalidad. Por razones de espacio no podemos exponer aquí las características de dichos modelos, información que esperamos publicar prontamente. No obstante, hemos presentado algunos de nuestros resultados en congresos recientes. Para más detalles consultar: Urmeneta, 2009, abril y junio, 26 a y b.
} 
Otros es una estructura que tiende repetirse en los textos escritos por los menores. Dicho antagonismo se construye en torno a dos grandes oposiciones: Uniformidad v/s Diferencia e Interior v/s lo Exterior.

En el primer caso, la identidad se construye en torno a un Nosotros cerrado, donde cualquier tipo de divergencia es rechazada y estigmatizada por el grupo. En estos cuentos, la identidad es entendida como uniformidad y en ella no hay cabida para la diversidad. Este modelo se halla presente principalmente en los estudiantes de escuelas católicas y en los de nivel socioeconómico alto. En la mayoría de los relatos correspondientes a este modelo, la persona diferente sufre un proceso de transformación, muchas veces penoso, tras el cual ésta conforma su conducta a los requerimientos de la norma y es reintegrada a la comunidad. El Otro es vinculado en los relatos infantiles a la ideas de mal (a veces como resultado de algún tipo de posesión maligna), incivilidad, irracionalidad, anormalidad (producto de un defecto físico, mental o de carácter). En casos extremos, el estigma de la diferencia, soportado por el sujeto transgresor, es de tal magnitud que no se da cabida en el relato a ningún tipo de final reconciliador: El personaje es expulsado para siempre o es condenado a muerte. El castigo en este caso es permanente, y no existe posibilidad de retorno a la norma y a la comunidad.

Suplicó que no le hiciesen nada y que se iría, pero ellos lo ataron a una hoguera junto con todas las otras víctimas. Gracias a eso aquella tribu pudo abonar las plantas con las cenizas del diferente Ambrosio. Esta historia está basada en hechos reales. (Hombre, 13 años, Escuela Laica, Nivel Socioeconómico Alto)

El segundo eje, basado en la oposición Interior/Exterior, es bastante recurrente en los relatos de los menores. La norma en este tipo de cuentos se encuentra claramente relacionada con la cadena asociativa: comunidad-nosotros-interior; mientras que la transgresión, se vincula a la secuencia opuesta: lo ajeno-otros-exterior. La norma es la base de la identidad de la comunidad, el sustento del Nosotros y lo que traza la frontera que separa lo de dentro de lo de fuera. El proceso de aprehensión e interiorización de la norma es representado en muchos relatos mediante un viaje de carácter iniciático (real o mágico/onírico); en el transcurso del cual el personaje protagónico comprende el sentido del Nosotros y el lugar que él o ella ocupa dentro de la estructura social. El viaje cumple una importante función dentro de la estructura del cuento: evidencia la tensión entre lo interno y lo externo, lo 
conforme a la norma y lo disidente. Los personajes protagónicos en este tipo de cuentos, experimentan un rito de paso (Van Gennep, 1985): transitan de un estado de separación, asociado a la transgresión, a un estado de agregación con la comunidad, asociado a la conformidad con la norma. Entre estos dos momentos del rito existe un espacio liminar o de margen, que en el caso de los relatos infantiles incluidos en este grupo, adopta la forma de un viaje iniciático. Es en este estadio liminar donde el personaje recibe los ritos apropiados para su posterior reintegración a la comunidad. El viaje, como rito de preparación para el paso a la agregación a la comunidad, comporta un elevado coste para el personaje principal, pues implica abandonar la seguridad proporcionada por el hogar familiar. Pero, también, supone una tentadora perspectiva, la posibilidad de recorrer el mundo, aunque esto le obligue a enfrentarse a los peligros del exterior.

[Mutombo, el protagonista] llegó a una ciudad, se ahogaba por culpa del aire que estaba un poco sucio por culpa de la contaminación, pero igualmente Mutombo continuo andando hasta dejar muy atras a lo que siempre habia sido su casa el gran bosque. Entonces unos niños vieron a Mutombo empezaron a reirse de cómo iba vestido, el pregunto que que pasava pero no respondieron entonces uno de los chicos lo agarro por detrás y le puso una navaja en el cuello el entonces se puso nervioso no sabia que hacer mientras los niños se reian de el. (Hombre, 14 años, Escuela Laica, Nivel Socioeconómico Bajo)

Algunos de los viajes narrados por los menores relacionan esta tensión a la oposición entre el campo y la ciudad. Mientras el mundo rural se vincula a la idea del hogar, la ciudad es definida en los relatos infantiles como el espacio exterior, el cual suele verse vinculado a las ideas de inseguridad, contaminación, drogadicción, suciedad y violencia. Los cuentos escritos por los menores indican que el personaje transgresor se siente como un extranjero en la urbe, y es precisamente esa experiencia de distancia la que le permite entender que se halla fuera de su referente normativo, y que para volver a la seguridad del hogar deberá acatar la norma de la comunidad.

Muchos de los textos analizados, definen la transgresión como el origen mismo de la destrucción y el caos, incluso se le asocia al exterminio de la comunidad. Del análisis de este tipo de textos, se desprende que para el imaginario infantil la violación de una norma particular supone la disolución del contrato social, y sus efectos se dejan sentir en toda la 
sociedad. Lo anterior indica la presencia en la infancia de una cierta comprensión monolítica de la norma, tema en el que ahondaremos en la siguiente sección.

\subsection{La Norma como Salvaguardia del Bien Común}

La segunda forma de representación de la norma se estructura en torno al eje Bien Común/Bien Individual. La norma protege la propiedad común, el beneficio de todos por sobre el particular. Hemos hallado dos grandes modelos respecto a cómo se representan la infancia la relación entre estos dos tipos de interés. En el primer modelo éstos son antagónicos, la transgresión es presentada como un conflicto entre el Bien Común y el Bien Individual. En el segundo, en cambio, ambos conceptos son complementarios: El Bien Individual se deriva del Bien Común, se trata de una relación de continuidad y no de ruptura como en el primer caso. Cada modelo se halla asociado a un cierto perfil de menores. El primero, se presenta con mayor claridad en los estudiantes de Escuelas Católicas; mientras que el segundo, predomina en las Escuelas Laicas. También el Género y el nivel socioeconómico parecen tener relación con esta distinción, aunque ésta resulta menos evidente que la hallada respecto a la ideología religiosa de las escuelas. Respecto al género, la mayoría de los niños se representa el Bien Común como algo opuesto al Bien Individual, mientras que las niñas visualizan ambos intereses de manera complementaria. En referencia a la variable nivel socioeconómico, los menores de estratos altos se representan el Bien Individual como una extensión del Bien Común, mientras que en los de estratos bajos encontramos ambos modelos: oposición y complementariedad.

En el primer modelo, de oposición entre Bien Común y Bien Individual, el interés personal es representado como un deseo mezquino. El personaje transgresor busca satisfacer su voluntad sin tener en cuenta las necesidades de los demás. Detrás de esta transgresión no hay necesariamente un objetivo claro. En muchas ocasiones, este afán egoísta es simplemente el producto de un capricho, sin que medie para ello una explicación racional. La norma en estos cuentos representa a la comunidad y es entendida como el origen primordial del Bien Común. Observamos en los textos infantiles una conocida dicotomía para las Ciencias Sociales: la oposición Individuo-Sociedad. La transgresión al estar asociada al interés personal, se vincula a la idea de individuo, mientras que la norma, como salvaguarda del Bien Común, es vinculada a la colectividad. Los relatos se centran precisamente en la 
narración de cómo y por qué el personaje transgresor decide renunciar a su interés individual, para someterse a los designios del Bien Común.

Otro aspecto de gran interés sociológico presente en los relatos analizados, es la creencia infantil en la existencia de un monismo o indisolubilidad de las normas. Esto indica que para el imaginario infantil la ruptura de una única norma supone la destrucción de todo el conjunto normativo. Los relatos argumentan, con bastante detalle, esta supuesta indisolubilidad de las normas y nos muestran cómo la transgresión conduce al caos anómico. Si la norma es el cimiento sobre el cual se construye la identidad colectiva, como se vio en el primer modelo, y si ésta constituye el sostén del Bien Común, como hemos visto en este segundo, la ausencia de normas, la anomia, supone una importante merma de la cohesión social e imposibilita cualquier tipo de contrato entre los individuos. Tal como se aprecia en el siguiente texto:

Ellos [sus amigos] le dijeron que si él podía ser malo porque los demas no podian ser. Saiwa salio corriendo y vio que el cartero cambiaba las cartas de sitio, el director tiraba globos de agua por la ventana, los abuelos escupian a los niños, etc. Saiwa Saiwa corrio al parque i recogio todo lo que estropeo y al verlo la otra gente empezo a pedir perdon y a arreglarlo todo, asi la ciudad volvio a su estado natural. (Hombre, 13 años, escuela católica, nivel socioeconómico bajo)

Este relato nos muestra un ejemplo de cómo para los menores las normas representan abstractamente el Bien, la garantía de que si tú eres bueno, todos los demás lo serán. La transgresión supone la extinción de dicha garantía. Cuando se rompe el lazo social que ata a los individuos al Bien, éstos son arrojados a la arbitrariedad de la anomia: si uno de ellos es "malo", todos tienen derecho a serlo, no hay nada que los obligue. El Contrato Social, y el orden y cohesión derivados de éste, se desmorona, al igual que todo el edificio social y el sistema de roles. La norma asegura que cada cual cumpla con su rol dentro de la sociedad. Los roles están asociados a la idea de expectativa, lo que se espera que haga cada individuo según su papel dentro del grupo. La transgresión al destruir él imperativo social, desintegra estas expectativas, esto conlleva a que todo se vuelva caótico e impredecible, tal como se describe en el cuento. En el Contrato Social, los individuos aislados encuentran la forma de organizarse, de constituirse en comunidad, y de avanzar todos juntos, bajo los principios de la norma, hacia el progreso y la construcción de un espacio mejor para todos y todas. La relación entre ambos tipos de bienes es claramente obligatoria, el Bien Individual debe 
supeditarse al Bien Común, éste es el principio general que subyace en este tipo de cuentos. Se espera del sujeto transgresor que retorne al orden, abrace la norma y supedite su interés individual al interés de toda la comunidad. Este es el comportamiento racional esperado de un "buen" miembro de la comunidad.

En el segundo modelo, en cambio, el Bien Individual es representado como la derivación del Bien Común. No hay contradicción entre estos intereses, por el contrario, su relación es de continuidad. Los relatos infantiles nos narran la historia de personajes que descubren que la oposición entre Bien Común y Bien Individual es sólo aparente, que no existe un conflicto entre ambos, y que es posible obtener un beneficio individual sin ir en contra de los intereses de la comunidad.

Cómodo salió como de costumbre al bosque a maltratar a todo ser vivo que veia. Cuando llego al poblado, a mediodia, para comer, Cómodo comenzo a encontrarse mal. [Era] una grave enfermedad, que solo se podia curar con unas plantas medicinales que se encontraban ya muy pocas por los alrededores ya que Cómodo las habia matado casi todas. .... Estando malo, Cómodo había podido pensar sobre su odio a la naturaleza. $Y$ pensó ¿si la naturaleza me ha salvado la vida, porque voy a odiarla? Cómodo, cada dia se iba al bosque, pero ya no iba para matar y destruir, si no para amar, respetar y cuidar al bosque. (Mujer, 13 años, Escuela Laica, Nivel Socioeconómico Bajo).

Si en el primer modelo, la estructura paralela de significados se construye en torno a la oposición entre Bien Común y Bien Individual, en este segundo modelo, la oposición fundamental es entre el Conflicto y la Conciliación de estos dos tipos de bienes. La transgresión es fruto de una contradicción aparente entre el Bien Común y el Bien Individual, el personaje transgresor descubre a lo largo del relato la verdadera naturaleza de la relación entre ambos bienes, la complementariedad. Detrás de este conflicto aparente, se oculta la lección que debe aprender el personaje: el Bien Individual emana del Bien Común. La norma es para los menores, la puerta de entrada a la inclusión social, es la clave de la integración que permite alcanzar el equilibrio entre Bien Individual y Bien Común. Este es el comportamiento racional que se espera del sujeto: ser capaz de conciliar el propio bien con el bien general, encontrar en el Bien Común el espacio adecuado para desarrollar, en armonía con la comunidad, el Bien Individual. Conformarse a la norma es un acto racional 
según se explicita en los relatos infantiles, en tanto que es ésta la que posibilita la continuidad entre ambos tipos de bienes: acatar la norma nos permite integrarnos en la comunidad y a través de ella satisfacer nuestras necesidades individuales en conciliación con las necesidades comunitarias.

\subsection{La Conformidad a la Norma como una Forma de Madurez}

La última de las formas de representación de la norma que hemos hallado en nuestro análisis, se basa en la idea de maduración. La conformidad es vinculada a las ideas de mayoría de edad, racionalidad, sensatez, responsabilidad y prudencia, las que hemos condensado en el concepto amplio de madurez. La norma se vincula al mundo de los mayores (aunque no necesariamente de los adultos). Es la experiencia adquirida con los años la que proporciona el discernimiento necesario para su comprensión. La norma otorga sentido a la acción de los sujetos, es la base racional que la fundamenta. El comportamiento infantil y juvenil, según los textos analizados, es irreflexivo, atolondrado, arbitrario, carente de sentido. El comportamiento de las personas adultas, o de los menores responsables que han alcanzado un cierto grado de madurez, es el que se ajusta a los dictados de la norma y, por tanto, a la definición social de lo racional.

Podemos clasificar los textos correspondientes a este modelo en tres grandes grupos, según la forma en que se representa la madurez y cómo ésta se asocia a la norma. En los dos primeros, el acatamiento de la norma es vinculado al género, al hecho de hacerse mujer y hacerse hombre; aunque la forma en que se construye la feminidad y la masculinidad difiere notablemente. En el tercer grupo, los conceptos más relevantes son la responsabilidad y la racionalidad; nos muestra el tránsito de un personaje que se comporta como un niño pequeño que hace lo que quiere, a un sujeto maduro que obedece la norma de la comunidad. Podemos ver cómo en los dos primeros modelos se halla presente la idea de edad; mientras que en el último, esta noción aparece difuminada, aunque sí encontramos con claridad la idea de paso del tiempo. El tránsito reflejado no es el de la niñez a la adultez, sino de un estado de desarrollo a otro dentro de una misma edad: se trata del paso del sujeto irresponsable e irracional a aquel que se somete a la norma, se comporta sensatamente y asume sus obligaciones sociales. En los tres casos nos encontramos ante diferentes formas de ritos de paso (Van Gennep, 1985) con una estructura similar a la que viéramos más arriba en los viajes iniciáticos. 
Al igual que en los casos anteriores, hemos analizado la relación de estos tres modelos con las variables género, nivel socioeconómico e ideología religiosa de la escuela. En términos generales, podemos señalar que la idea de la conformidad a la norma como una forma de madurez se halla presente con mayor claridad en los estudiantes de escuelas católicas que en los de escuelas laicas, con independencia de su extracción social. Esta forma de representación de la norma se halla prácticamente ausente en los estudiantes de escuelas laicas. También la variable género ocupa un papel destacado, los cuentos de chicos y chicas son diferentes.

Los textos comprendidos en el primer tipo tienen un elemento claramente común: son cuentos escritos por niñas que narran historias de personajes femeninos. No obstante, en el marco general de la muestra, y no ciñéndonos únicamente a los textos incluidos en este modelo, éste no es un tipo de cuento predominante entre las mujeres: los cuentos con protagonistas femeninos representan sólo un $28 \%$ del total de la muestra $(n=896)$. Sin embargo, desde una perspectiva cualitativa, este tipo de relatos es especialmente relevante. En muchos de estos textos se produce una curiosa contradicción: si bien podrían considerarse como cuentos de resistencia, en tanto que constituyen una alternativa a la hegemonía masculina presente en la mayoría de casos, son notablemente reproductores respecto a los roles de género y a la forma en que se representa la feminidad.

La transgresión es entendida por las niñas como una forma de anormalidad. El personaje se halla fuera de su estado natural y de las condiciones que le son inherentes. En el texto que presentamos a continuación, podemos ver cómo la protagonista tiene una conducta que se distancia de la definición social de lo femenino. Su comportamiento es doblemente transgresor: atenta contra la ley de la comunidad y contra la "naturaleza" de su condición de mujer.

Se enamoró de un chico .... Lara nunca pensó que el chico le fuera a ser pasar por la cabeza el canviar de personalidad y de aspecto, Lara pensó que si camviaba de personalidad y aspecto a lo mejor podia gustar a este chico, ella canvio empezó a vestir con una chica normal y no seguir como antes con chandals sin peinar con nudos en el pelo etc. Se le podría decir que era un machote pero ahora según ella se dedicaría a cuidar su cuerpo y ser normal. (Mujer, 13 años, escuela católica, nivel socioeconómico bajo). 
Encontramos en este cuento, como en los relatos incluidos en este modelo, abundantes referencias a la idea de normalidad v/s anormalidad. Se vincula la violación de la norma de la comunidad al abandono de la propia esencia. Esta relación tiene un carácter recursivo: la transgresión de la norma se explica por la desnaturalización de la concepción "normal" de feminidad, la que a su vez es producto de la ruptura de la norma. Podemos ver reflejada la asociación entre estas dos formas de transgresión en las ideas de "personalidad y aspecto", que la autora menciona repetidas veces en su relato. La protagonista tiene una personalidad y un aspecto inadecuado, que no calza con el molde socialmente construido, e impuesto, de normalidad para las mujeres. Estos dos elementos actúan en forma conjunta dentro del relato, retroalimentándose mutuamente y formando una suerte de círculo vicioso, el cual queda plasmado en el texto en la figura del "machote".

En el segundo modelo, en cambio, no encontramos en el discurso infantil una insistencia tan marcada sobre la masculinidad, como la que vimos en el primero respecto a la feminidad. Ésta se halla presente, aunque de una forma menos evidente. Los textos correspondientes a este tipo, comparten una característica común, son cuentos escritos por niños que narran la historia de protagonistas masculinos. La transgresión es presentada como un conflicto con la autoridad del padre (no encontramos referencias a la madre en este sentido). Se trata de niños o adolescentes que quieren hacerse mayores, que desean convertirse en hombres y recorrer el mundo, pero que se ven atados al hogar, una barrera que difícilmente pueden superar. El siguiente texto nos narra la historia de un chico adolescente que, a través de la transgresión, busca oponerse a la potestad del padre.

Se fugó de casa porque no le dejaron cazar insectos su padre, entonces Chichi hizo las maletas y se fue al pueblito de arriba que está a seis kilómetros de su pueblito .... fue a ver a un amigo mayor que se llamaba Garogendo que tiene 17 años. Garogendo le dijo que cómo había hecho eso, entonces Chichi le contestó porque en mi casa no se puede hacer nada, entonces Garogendo le dijo que si quería quedarse a dormir .... al día siguiente fue la policía a casa de Gerogendo para preguntar por Chichi .... entonces a Gerodendo lo detuvieron y entraron a la casa de Gerogendo y descubrieron que tenía muchas drogas, entonces Chichi se fue porque tenía droga, entonces los padres de Chichi tuvieron mucho miedo porque se pensaban que se había llevado droga pero eso no es así Chichi era sano como una rosa, Chichi se fue a casa de su tío y le explicó lo que había pasado con su padre entonces su tío llamó a su padre y les 
dijo que Chichi estaba en su casa. Entonces el padre fue a la casa del hermano y cogió al Chichi y se lo llevó para casa. (Hombre, 14 años, escuela católica, nivel socioeconómico bajo). [Nota: Traducido del catalán].

En este cuento podemos ver la lucha de un adolescente por convertirse en mayor, en hombre; estructura que se repite en todos los textos incluidos en este modelo. Comienza la aventura con el protagonista huyendo de su hogar iniciando un viaje de carácter iniciático, de características similares a las del que hemos descrito más arriba. Nuevamente, encontramos la oposición entre el hogar, donde destaca la figura del padre, y mundo exterior, asociados respectivamente a las ideas de niñez y adultez. El mundo interior, el hogar, representa lo conocido, la seguridad, el bien. El mundo exterior, en cambio, se asocia a la figura contraria. Se trata del mundo ignoto, un terreno por recorrer, potencialmente peligroso y maligno, pero siempre atractivo.

La concepción negativa del mundo exterior se ve plasmada en este cuento con bastante claridad en la figura del amigo mayor, que oculta en su casa al protagonista. Este personaje se nos presenta como una figura oscura, la encarnación de dicho mundo, que amaga tras la apariencia de una vida normal un turbio negocio de tráfico de drogas. También representa los peligros que comporta el hacerse mayor y el abandono del seguro y puro mundo infantil. La figura del amigo delincuente se opone a la del protagonista "sano como una rosa", preservado, hasta ahora, de la perversión adulta. Su función dentro del relato es propiciar su contacto con este mundo sombrío. El contraste entre lo interno y lo externo, permite discernir al personaje cuál es el camino correcto, retornar a la norma y a la tutela del padre. Es precisamente, la experiencia vivida en esta oscuridad, la que le permite reconocer la luz y abrazarse definitivamente a ella. Se trata de una visión notablemente estereotipada, sin matices, en la que "todo lo bueno" es asociado al mundo familiar y "todo lo malo" al mundo exterior.

Hacia el final del relato, encontramos la figura del tío que cumple el papel de mediador entre los padres y el protagonista. El tío, una figura adulta, segura y limpia, representa un lugar intermedio, que permite completar el retorno a la norma del personaje. El tío actúa como un puente entre los dos mundos, permitiendo al protagonista diferenciar la luz y la oscuridad que representan las otras figuras. El tío obliga al protagonista a volver a la realidad, aceptar 
lo que es, un niño, y acatar la autoridad paterna. Finalmente, el cuento se cierra con los padres llevando físicamente al protagonista de vuelta al hogar, lo que puede ser entendido como una representación del retorno del a la norma. Es importante destacar que todas las figuras que aparecen en el relato, tanto las positivas como las negativas, son masculinas; un indicio más de que el tema central del cuento es la masculinidad.

Podemos ver cómo este tipo de cuento se distancia notablemente de los escritos por las niñas. Nos encontramos ante un modelo, que si bien se centra en la idea de la edad y en la conformidad a la norma como una forma de madurez, recibe connotaciones muy distintas a las que hallamos en el caso de las chicas. Los niños narran la oposición a la autoridad que realizan personajes masculinos para reafirmar su propia identidad. Se trata de un intento por crecer, por ser mayor, por convertirse en hombres. Sin embargo, estos intentos se nos presentan como fallidos: los niños siguen siendo niños, aunque ahora con el conocimiento y la aceptación de esa realidad. Las niñas, por su parte, nos narran un tipo de historia distinto. Nos cuentan la aventura de personajes femeninos que buscan, al igual que sus homólogos masculinos, crecer, hacerse mayores, convertirse en mujeres. Sin embargo la maduración en hombres y mujeres tiene un carácter diferente: mientras en los niños se relaciona con la rebelión y la reafirmación del propio yo; para las niñas, hacerse mujer, supone más bien amoldamiento que oposición. La maduración tiene que ver con el descubrimiento de la propia feminidad. Pero, no se acepta cualquier tipo, sólo se considera femenino lo que se ajusta al patrón social de mujer. Otras formas alternativas son tratadas como anormalidades o desviaciones del modelo hegemónico. A diferencia de sus pares masculinos, las protagonistas femeninas tienen éxito en su lucha por convertirse en mujeres. Alcanzan la madurez e incluso la adultez, hecho que queda plasmado en los relatos en las referencias al matrimonio, los hijos y en general a la idea de formar una familia, actos que coronan su proyecto de feminidad.

El tercer y último modelo incluye relatos en los que se desarrollan temas bastante diferentes a los expuestos hasta aquí. No se trata de relatos centrados en la feminidad, ni en la oposición a la autoridad como una forma de probar la hombría; sino en el acatamiento de la norma como una forma de responsabilidad y racionalidad. La narración nos relata el proceso mediante el cual el personaje transgresor, generalmente definido como una persona inmadura o atolondrada, logra comprender el sentido de la norma y las consecuencias que 
conlleva su transgresión. En el desarrollo del relato, este sujeto adquirirá cordura, aprenderá a hacerse responsable de sus actos y a actuar con sensatez; cambios que se expresan en el texto mediante la conformidad con la norma.

Este modelo se concentra en los estudiantes de nivel socioeconómico alto. No hemos hallado en el análisis cualitativo (realizado en una submuestra de 150 casos) cuentos de este tipo entre los estudiantes de nivel socioeconómico bajo. La variable género no es tan importante como en los modelos anteriores, encontramos tanto casos de chicos como de chicas, aunque predominan éstas últimas. Respecto a la variable ideología religiosa de la escuela, predominan con rotunda claridad los estudiantes de escuelas católicas. En el texto siguiente el transgresor es presentado como un chico irracional, que es expulsado de la comunidad hasta alcanzar la edad adulta:

Se le acercó Rumí, uno de los jefes de la comunidad y le dijo: -Filip, el Gran Padre te quiere ver, deprisa. Filip se levantó y con el miedo en el cuerpo, ya que le temblaban las piernas se fue a hablar. [El Gran Padre] era considerado dentro de la comunidad el ser supremo, el magnífico y todo lo que él decía se debía aceptar. .... El Gran Padre le dijo:-Filip, como un miembro de los babalins has desobedecido mis normas y has destrozado parte de nuestras tierras. Como castigo deberás ser expulsado de la tribu y nomás podrás regresar hasta cuando seas adulto.-¡Viva!- Pensó Filip, ya que él no se daba cuenta del futuro que le esperaba, duro pero muy provechoso. .... Con el paso de los años se convirtió en un auténtico escritor conocido en todo el país. Formaba parte de un grupo ecologista, ya que su carácter destructivo y antinatural se había convertido con el tiempo en un completo defensor de la naturaleza, la cual defendía aunque no le agradaba para vivir. El día en que cumplió 24 años, edad con las que los babalins consideraban que una persona ya era adulta decidió volver a la comunidad, se sentía orgulloso de haber nacido ahí y quería agradecer al Gran Padre todo lo que había hecho por él, ya que sus conocimientos y su inteligencia eran gracias a la ayuda que el Gran Padre le había proporcionado durante su infancia. De eso ya han pasado unos cuantos años y antes de llegar a ser demasiado viejo para hacerlo Filip escribió y explicó su vida en un libro titulado "Memorias de un babalí" donde explicó por qué luchó y como su sueño se hizo realidad. (Mujer, 14 años, escuela católica, nivel socioeconómico alto). [Nota: Traducido del catalán. Cuento titulado: Memorias de un Babalí]. 
La comunidad, representada por su máximo exponente, el Gran Padre, interpela directamente al transgresor. La autoridad del jefe de la tribu, ser supremo y magnífico, es inapelable, todo lo que él dicta se ha de obedecer, sin objetar ni rechistar. El protagonista espera temeroso el dictamen del anciano, quien decide expulsarlo de la tribu hasta que alcance la adultez. La transgresión en este cuento aparece asociada con bastante claridad a la idea de inmadurez. Filip es incapaz de comprender el sentido de la norma, aún no pertenece en propiedad a la comunidad y debe ser iniciado para participar de pleno derecho en ella. Éste es precisamente el papel que cumple el viaje en el cuento ${ }^{8}$.

El personaje principal logra una vida exitosa en su exilio: tiene un buen trabajo, ha adquirido fama y se ha convertido en un ecologista, signos que ponen de manifiesto su nuevo grado de madurez. La autora nos resume muy bien el cambio experimentado por el protagonista: "su carácter destructivo y antinatural se había convertido con el tiempo en un completo defensor de la naturaleza". Tal como había vaticinado el Gran Padre, el tiempo ha cumplido su cometido y con la llegada a la adultez el protagonista ha adquirido la consciencia y sensatez de la que carecía en su juventud. Una vez completado el ciclo de maduración, representado en el relato por la edad de la adultez, los 24 años, éste decide retornar a su hogar. Se trata del anunciado regreso del hijo pródigo al hogar.

Finalmente, el personaje transgresor retorna a la norma. El viaje iniciático ha permitido al protagonista encontrar su propia identidad, y con ella la madurez requerida para integrarse en forma plena a la comunidad. El cuento se cierra con su conversión: la transformación del transgresor en un paladín de la norma. El protagonista, no sólo regresa a su antiguo hogar convertido en un escritor famoso y en un denodado ecologista, sino también trasciende los límites temporales y espaciales del texto al transformar sus memorias personales en el cuento que ahora leemos: Memorias de un Babalí.

En general, los cuentos incluidos en este tercer modelo, donde el origen de la transgresión es asociado a la inmadurez del personaje protagónico, ofrecen elaboradas justificaciones de la norma. Se enfrenta la irracionalidad del sujeto transgresor a la racionalidad de la

\footnotetext{
${ }^{8}$ Un viaje iniciático de características similares a las reseñadas en los casos anteriores, que no hemos reproducido aquí por motivos de espacio.
} 
comunidad encarnada en la norma; subyace en esta contradicción la oposición individuosociedad, que viéramos en anteriores cuentos. Los motivos expuestos por la infancia sobre la necesidad de acatar la norma son variados, pero podemos agruparlos en dos grandes categorías: las razones instrumentales y las razones sustantivas o referidas a valores (Weber, 1984). Desde la perspectiva instrumental, la norma debe ser acatada porque es útil y presta un servicio importante a la comunidad. En este tipo de cuentos, se utiliza con frecuencia la idea de Bien Común: la norma garantiza el interés general, representado en el cuento por los parques y bosques. Desde la perspectiva sustantiva, en cambio, la norma es fundamentada por la tradición y los valores de la comunidad, como sería el caso del cuento analizado antes. La norma aparece asociada a la idea del Nosotros, debe ser respetada porque resguarda nuestras costumbres, historia e identidad como comunidad.

La transgresión es entendida en los tres tipos analizados aquí, como un acto propio de la inmadurez e ignorancia del personaje principal, y no como una afrenta a la comunidad. Se trata de un atentado contra sí mismo, ya que el máximo perjudicado de esta acción es el propio sujeto. Más allá de las particularidades de cada modelo, todos los textos muestran algunos rasgos comunes que creemos importante destacar. En todos los casos, la madurez y el paso del tiempo ocupan un papel central, esto es lo que permite resolver el conflicto generado por la transgresión. La conformidad a la norma es asociada en algunos cuentos a las ideas de masculinidad y feminidad, y en otros, a las ideas de racionalidad y responsabilidad. Otro aspecto común en este tipo de textos es la importancia que se asigna al núcleo familiar del personaje. Si en otros relatos encontrábamos diferentes tipos de agentes, en este modelo la presencia de familiares o amigos cercanos es claramente mayoritaria. Son éstos los que acompañan y propician el cambio del protagonista, o simplemente son testigos privilegiados del mismo.

\section{Conclusiones}

Introducirse en el mundo social infantil es en sí mismo un desafío sorprendente y enormemente enriquecedor. Es un trabajo arduo, pero sin lugar a dudas maravilloso. Impresionan profundamente sus anhelos de justicia social, su preocupación por la guerra, el hambre, la violencia, la discriminación, la intolerancia y un sinfín de problemas sociales, que se ven claramente reflejados en su discurso. En algunas ocasiones sus relatos pueden dejarnos con un cierto sinsabor: la profundidad y acidez de sus críticas a nuestra sociedad, 
lo grises e hipócritas que nos ven como adultos. Sus curiosas ocurrencias y desbordante imaginación, ciertamente nos pueden hacer sonreír.

Sus cuentos nos permiten asomarnos a lo que ellos y ellas entienden como justo, normal y racional, y sus respectivos contrarios. Nos proporcionan pistas de cómo creen que funciona el mundo social, cuáles son sus principales instituciones y actores y qué papel en el gran engranaje social ocupa cada uno de ellos. A través de los relatos podemos pasar del nivel individual y microsocial al nivel macrosocial: desde cómo se conciben a sí mismos y su entorno más próximo, a cómo se representan la sociedad en general, especialmente en lo referente a la norma y la transgresión. La técnica Cuento Inconcluso constituye una verdadera mina de oro: ¡Tenemos ante nuestros ojos la visión infantil sobre la sociedad!

Los resultados presentados en este artículo muestran algunas de las imágenes sociales que subyacen en los textos producidos por los menores. Son un indicativo de la concepción y la tolerancia que tienen respecto al Otro, de ahí la radical importancia de seguir estudiando esta problemática. En sociedades cada vez más complejas y multiculturales como las nuestras, resulta imprescindible conocer el contenido de las representaciones sociales infantiles, para detectar y trabajar de manera activa en los estereotipos presentes en su discurso, y para procurar la reflexión y la construcción de un conocimiento colectivo que permita integrar e incluir la diferencia.

Los resultados de la técnica Cuento Inconcluso son realmente prometedores. Se trata de una herramienta de investigación, pero también de acción, que puede ser utilizada no sólo con fines académicos, sino también para la transformación educativa. Los datos proporcionados por esta técnica pueden ser empleados como guía por las personas educadoras en procesos de investigación-acción o de intervención socioeducativa. Ofrecemos a continuación algunas posibles líneas de acción y análisis, que pueden ser abordadas en futuros estudios.

1) Tratamiento de la diversidad: el análisis de la representaciones sociales infantiles sobre la norma permite comprender cómo la infancia construye su noción del Nosotros y su contrario, los Otros. Resulta especialmente importante trabajar con ellos y ellas la distinción que realizan entre uniformidad y diversidad, así como sus concepciones de la norma y la transgresión. También, consideramos relevante ahondar en su 
representación sobre la ciudad, un lugar que en muchos cuentos es percibido como amenazante; más aún teniendo en cuenta que los participantes de nuestro estudio provienen de zonas urbanas.

2) Concepción del Bien Común: es preciso estudiar en mayor profundidad la representación infantil de la norma como sostén del interés general, ya que de este análisis podemos obtener una imagen detallada de cómo la infancia comprende la relación individuo-sociedad. Creemos fundamental seguir explorando sus concepciones del Bien Común y el Bien Individual. Queda pendiente por explicar por qué algunos menores definen estos conceptos como opuestos (especialmente, los estudiantes de escuelas católicas y los varones) y otros, en cambio, los entienden como complementarios (estudiantes de escuelas laicas y mujeres).

3) Definición de los Roles de Género: el imaginario social infantil se halla fuertemente influenciado por un patrón tradicional de roles de género. Además de encontrar una clara hegemonía masculina en la mayoría de relatos, los escasos cuentos protagonizados por personajes femeninos son notablemente reproductores respecto a los roles de género y a la forma en que se representa la feminidad. Consideramos fundamental ahondar en esta temática y buscar posibles explicaciones al hecho de que los chicos construyan su masculinidad como rebelión contra la autoridad paterna; mientras que las chicas comprendan su feminidad como un proceso de amoldamiento a un patrón social que fija los atributos que conforman la naturaleza de la mujer y define como desviación cualquier forma alternativa.

Es muy importante destacar que nuestros resultados revelan la existencia de un pensamiento sociológico infantil. Hecho que nos permite afirmar con bastante contundencia que la infancia posee una mirada activa sobre su mundo social, que piensa, analiza y crea sus propias hipótesis sobre cómo funcionan las instituciones sociales y la sociedad en la que se hallan insertos. Concluimos, por tanto, que la infancia no puede seguir siendo considerada como un sujeto pasivo, mero objeto o recipiente, ni se puede negar o restar su potencial de acción. Los menores no son seres pre-sociales o agentes sociales incompletos. Tienen sus propias formas de interpretar, construir e influir de manera activa en su mundo 
social. Nuestra intención en este estudio es sentar las bases para el desarrollo de una sociología de la infancia que sitúe realmente a ésta en el centro de la investigación.

El estudio empírico de las representaciones infantiles es fundamental, pues proporciona elementos para el análisis y el debate, que pueden ser útiles tanto para la investigación como para la escuela y las familias. La técnica Cuento Inconcluso puede ser adaptada para el estudio de objetos sociales diversos. Puede abrirnos las puertas al conocimiento de las representaciones infantiles sobre los roles de género, la familia, los grupos sociales minoritarios (inmigrantes, homosexuales, minorías étnicas, etc.) y en forma general de cualquier tipo de problemática social o escolar. Incluso, puede proporcionarnos pistas sobre cómo están pensando los menores al otro, en fenómenos tan complejos y preocupantes como el bullying o la homofobia.

Consideramos que estos resultados pueden contribuir a la construcción de una escuela liberadora, que integre e incluya a los niños y niñas de origen diverso o minoritario, y que capacite a sus estudiantes para pensar y cuestionar de manera activa y crítica sus visiones sobre el Nosotros y los Otros. Otorgamos tal centralidad a la escuela, puesto que ésta constituye un lugar de encuentro obligado entre estudiantes de diferente condición sociocultural. De ahí que los discursos que ésta produzca sobre este tema sean especialmente importantes; no sólo en cuanto a su influencia en la producción de las representaciones sociales infantiles sobre la norma, la transgresión y en forma general, del Otro, sino porque entendemos que la escuela constituye un espacio social privilegiado para la inclusión/integración de la diferencia desde una perspectiva multicultural. Lo anterior pasa necesariamente por: un reconocimiento de la relatividad de los discursos transmitidos por la escuela y de su imposible neutralidad, y por la revelación de los estereotipos que el discurso social de ésta y otros actores sociales sostiene sobre determinados colectivos. Un discurso que puede permear y de hecho permea el pensamiento social infantil.

El estudio de su imaginario social debería ser complementado con el análisis de las representaciones sociales de los actores sociales que interaccionan cotidianamente con los menores (docentes, padres y madres, autoridades educativas y las personas investigadoras) y de las ideologías que subyacen en los discursos a los que se ve sometida la infancia (discursos provenientes de muy diversas fuentes tales como la escuela, los libros de texto, 
los medios de comunicación, los videojuegos, las organizaciones políticas y los discursos de los expertos, entre otros).

Pensamos que la escuela debe proporcionar a los estudiantes las herramientas que les permitan aprehender la realidad desde una perspectiva crítica. La educación debe capacitar al menor para que éste tome conciencia de que existe una diversidad de mundos posibles y para que imagine y construya otros mundos alternativos. La educación debe tratar de ser una fuerza eminentemente desreificadora. Las ciencias sociales, y específicamente la sociología como disciplina humanística, pueden y deben ocupar un papel destacado en la conformación de una nueva forma de comprender ese proceso de construcción social de conocimiento que llamamos educación.

\section{Referencias}

Arias, María Mercedes. (1999). Triangulación metodológica: sus principios, alcances y limitaciones. Recuperado en Julio de 2005, de http://www.robertexto.com/archivo9/triangul.htm

Berger, Peter. (2002). Introducción a la Sociología. México: Limusa.

Berger, Peter \& Luckmann, Thomas. (1999). La construcción social de la realidad. Buenos Aires: Amorrortu.

Bericat, Eduardo. (1998). La integración de los métodos cuantitativo y cualitativo en la investigación social. Barcelona: Ariel.

Durkheim, Émile. (1982). Las Formas Elementales de la Vida Religiosa. El Sistema Totémico en Australia. Madrid: Akal.

Durkheim, Émile. (2001). La división del trabajo social. Madrid: Akal.

Farr, Robert M. (1983). Escuelas europeas de psicología social: la investigación de las representaciones sociales en Francia. Revista Mexicana de Sociología , 45 (2).

Gergen, Kenneth. (1993). El movimiento del construccionismo social en la psicología moderna. Sistemas familiares (2).

Greïmas, Algirdas Julius. (1983). La semiótica del texto: ejercicios prácticos. Análisis de un cuento de Maupassant. Barcelona: Paidós. 
Hiernaux, Jean Piere. (1977). L'Institution Culturelle. Méthode de description structurale. [La Institución Cultural. Método de descripción estructural]. Lovaina: Presses Universitaires de Lovain-UCL.

Hillmann, Karl-Heinz. (2001) Diccionario enciclopédico de sociología. Barcelona: Herder.

Jenks, Chris. (1982). The Sociology of Chilhood. Esential readings. [La sociología de la infancia. Lecturas esenciales]. Londres: Greggs Revivals.

Kohlberg, Lawrence. (1992). Psicología del desarrollo moral. Bilbao: Desclée de Brouwer.

Kohlberg, Lawrence; Power, F. Clark; Higgins, Ann. (2008). La educación moral según L. Kohlberg. Barcelona: Gedisa.

Moscovici, Serge. (1985-1986). Psicología Social. Barcelona: Paidós.

Propp, Vladimir. (1985). Morfología del cuento y Las transformaciones de los cuentos maravillosos. Madrid: Fundamentos.

Remy, Jean \& Ruquoy, Danielle. (Eds.). (1990). Méthodes d'analyse de contenu et sociologie. [Métodos de análisis de contenido y sociología]. Bruselas: Facultés Universitaires Sant-Louis.

Rodríguez Pascual, Iván. (2006). Redefiniendo el trabajo metodológico cualitativo con niños: el uso de la entrevista de grupo aplicada al estudio de la tecnología. EMPIRIA. Revista de Metodología de Ciencias Sociales (12), 65-88.

Sills, David L. (Dir.) (1979). Enciclopedia Internacional de las Ciencias Sociales. Madrid: Aguilar.

Thërborn, Göran. (1987). La ideología del poder y el poder de la ideología. Madrid: Siglo $\mathrm{XXI}$.

Urmeneta, Ana Rebeca. (2004). La Inmigración vista por los Niños y las Niñas. Investigación exploratoria en la ciudad de Barcelona. En Actas de la I Reunión Científica Internacional sobre Etnografía y Educación, Talavera de la Reina. Valencia: Germania.

Urmeneta, Ana Rebeca. (2008). Reificación, Reproducción y Resistencia al Mensaje Disciplinario de los Cuentos Infantiles. Tesis doctoral para optar al grado de Doctora en Sociología. No publicada. Universidad de Barcelona, Barcelona, España.

Urmeneta, Ana Rebeca. (2009, abril). Anormals, incívics i irracionals. Representacions socials infantils sobre la transgressió. [Anormales, incívicos e irracionales. Representaciones sociales infantiles sobre la transgresión]. Trabajo presentado en el $\mathbf{V}$ Congrés Català de Sociologia. Barcelona, España.

Urmeneta, Ana Rebeca. (2009, junio 26, a). El cuento inconcluso. Una herramienta innovadora para el estudio de las representaciones sociales infantiles. Trabajo 
presentado en el XIV Congreso Nacional de Modelos de Investigación Educativa. Asociación Interuniversitaria de Investigación Pedagógica AIDIPE, Huelva, España.

Urmeneta, Ana Rebeca. (2009, junio 26, b).Buenos y malos ¿Cómo se representan los niños y las niñas las normas sociales?. Trabajo presentado en el XIV Congreso Nacional de Modelos de Investigación Educativa. Asociación Interuniversitaria de Investigación Pedagógica AIDIPE, Huelva, España.

Van Gennep, Arnold. (1985). Los ritos de paso. Madrid: Taurus.

Weber, Max. (1984). Economía y sociedad. Esbozo de sociología comprensiva. México: Fondo de Cultura Económica. 